\title{
A evolução das importações entre 1999 e 2008: uma análise comparativa entre Brasil e Paraná
}

Luciano Nakabashi*

Marcio José Vargas da Cruz ${ }^{* *}$

RESUMO - Este artigo trata da evolução do valor, quantidade e origem das importações nas esferas nacional e estadual (Paraná) entre 1999 e 2008. Além de analisar a evolução geral é também apresentada uma análise desagregada para a economia paranaense das variações dos itens que compõem a pauta de importações. Por fim, o artigo apresenta perspectivas de mudança no desempenho das importações em função da desaceleração da economia, queda dos preços das commodities e desvalorização da taxa de câmbio.

Palavras-chave: Importações. Brasil. Paraná.

\section{INTRODUÇÃO}

O desempenho do setor externo de uma economia propicia leituras muito interessantes em relação à estrutura produtiva e ao efeito do comportamento de variáveis macroeconômicas, como renda e taxa de câmbio, nas transações com o resto do mundo.

De acordo com os dados das Contas Nacionais (IBGE), disponibilizados pelo IPEADATA (2008), a taxa média de crescimento da economia brasileira entre 2004 e 2007 foi de 4,63\%. Considerando a média-móvel de quatro anos e comparando com os demais períodos, desde a década de 1980, essa taxa foi menor apenas nos períodos compreendidos entre 1984-87 e 1985-88. Se a taxa de crescimento em 2008 for de, aproximadamente, 5,6\%, conforme as expectativas de mercado apresentadas pelo relatório Focus (Banco Central do Brasil, 2008), então a média quinquenal (2004 -2008) será de, aproximadamente, 4,82\%, sendo que essa média fica atrás apenas da média do período 1984-88 (4,84\%), considerando todas as médias quinquenais, desde o início dos anos 1980.

Além do ciclo de expansão econômica recente, o país também apresentou um processo contínuo de valorização da taxa de câmbio, desde o início de 2003 até setembro de 2008, o qual foi revertido com o cenário da recente crise financeira internacional.

A combinação de expansão econômica com o processo observado de valorização da taxa de câmbio $^{41}$ tende a resultar em fortes estímulos à importação, comportamento já

\footnotetext{
* Doutor em Economia pelo CEDEPLAR/UFMG. Coordenador do boletim Economia \& Tecnologia e professor do Departamento de Economia (DEPECON-UFPR). Endereço eletrônico: luciano.nakabashi@ufpr.br.

** Mestre em Economia pela Universidade Federal do Paraná. Professor do Departamento de Economia (DEPECON-UFPR). Endereço eletrônico: marciocruz@ufpr.br.
} 
previsto por diversos modelos macroeconômicos para economias abertas. Apesar dos indícios de alteração no comportamento destas variáveis, em função da atual crise financeira (há uma tendência a uma queda no ritmo de crescimento da economia brasileira e de desvalorização da taxa de câmbio).

Diante deste cenário, o presente artigo trata da evolução do valor, quantidade e origem das importações nas esferas nacional e estadual (Paraná) entre 1999 e 2008. Além de se analisar a evolução geral, também se realiza uma análise para a economia paranaense das variações dos itens que compõem a pauta de importações e suas perspectivas de mudança no médio prazo.

\section{O DESEMPENHO DAS IMPORTAÇÕES NO PARANÁ E NO BRASIL}

As importações paranaenses e brasileiras vêm aumentando de forma significativa, desde 2004, como podemos ver no Gráfico 1 (valores para o Paraná no eixo esquerdo e para o Brasil no eixo direito). Os valores são as médias mensais de cada ano e estão deflacionados pelo Índice de Preço do Atacado dos Estados Unidos em valores de setembro de 2008.

Os principais fatores que explicam esse desempenho são o crescimento da economia nacional e a apreciação da taxa de câmbio, até meados de setembro deste ano. O crescimento médio mensal do valor real das importações paranaenses foi de 193\%, enquanto que das importações brasileiras foi de 167\%, entre 1999 e 2008.

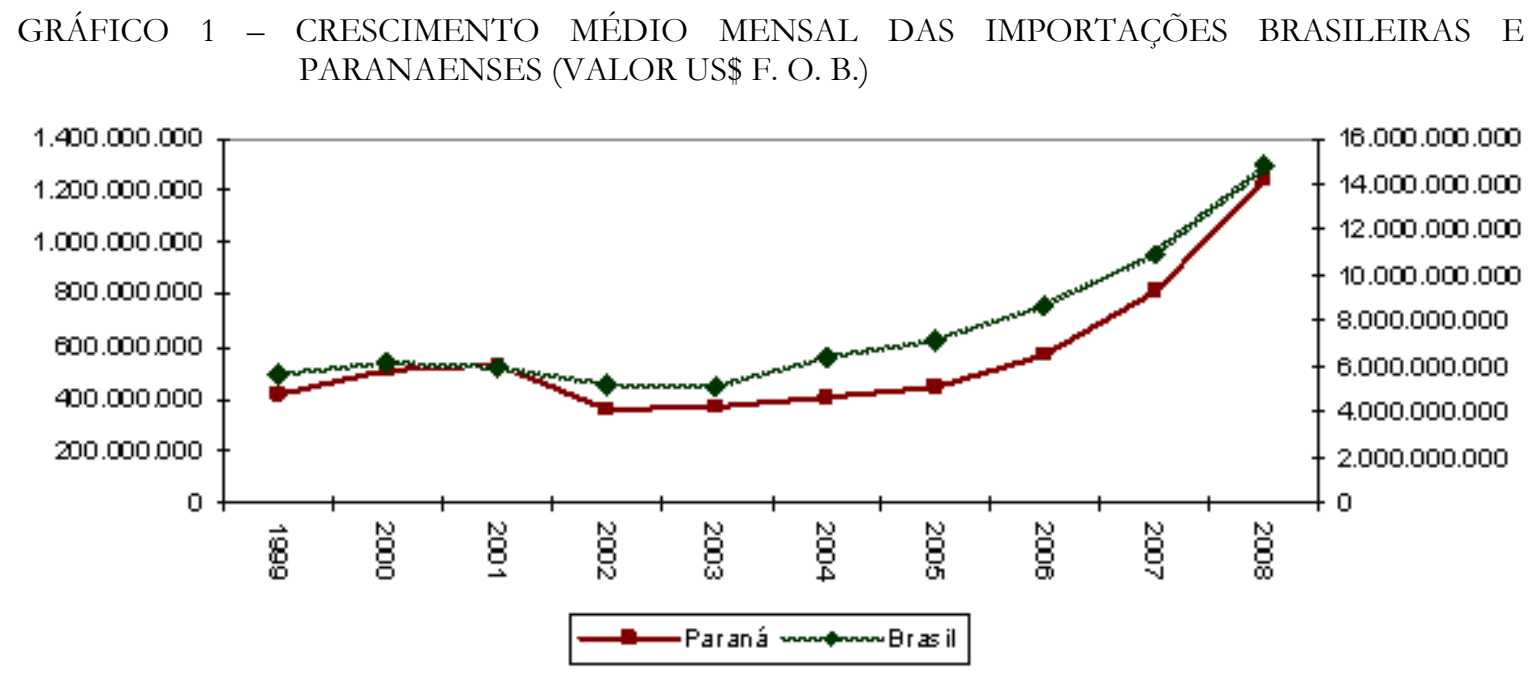

FONTE: Elaboração própria a partir de dados do SECEX/MDIC

O Gráfico 2 apresenta o crescimento médio mensal das importações em $\mathrm{Kg}$ líquido, ou seja, quantidade (valores para o Paraná no eixo esquerdo e para o Brasil no eixo direito). O crescimento da quantidade foi consideravelmente menor do que o do valor. O crescimento

41 Os gráficos que apresentam o comportamento da taxa de crescimento e da taxa de câmbio para a economia brasileira entre 1998 e 2008 estão em anexo.

92 
médio mensal da quantidade (em $\mathrm{Kg}$ líquido) das importações paranaenses foi de 61\%, enquanto que das importações brasileiras foi de 49\%, entre 1999 e 2008.

\section{GRÁFICO 2 - CRESCIMENTO MÉDIO MENSAL DAS IMPORTAÇÕES BRASILEIRAS E PARANA- ENSES (KG LÍQUIDO)}

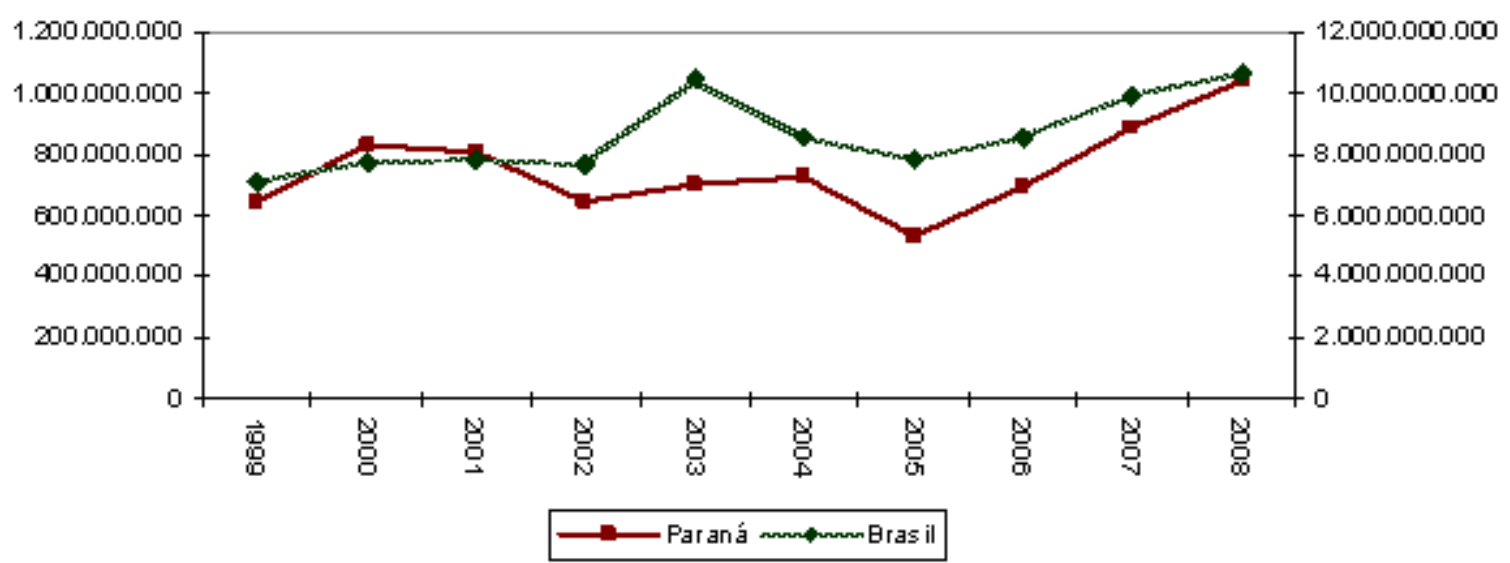

FONTE: Elaboração própria a partir de dados do SECEX/MDIC

Esse comportamento implica que houve um efeito significativo do aumento real dos preços das importações brasileiras e paranaenses, no período em consideração. Esse efeito preço reflete a elevação da demanda mundial por insumos industriais, inclusive do Brasil, devido ao elevado crescimento da economia mundial e brasileira no período. Desse modo, vem ocorrendo um aumento dos preços desses produtos, em dólares. Esse efeito é, parcialmente, compensado pela apreciação da taxa de câmbio.

A taxa de crescimento do Brasil e dos estados brasileiros é apresentada na Tabela 1. Nela, fica evidente a aceleração do crescimento da economia brasileira a partir de 2004, assim como da economia de vários estados brasileiros.

\section{ORIGEM DAS IMPORTAÇÕES BRASILEIRAS E PARANAENSES}

Em relação à origem das importações, pelo Gráfico 3, podemos notar a queda relativa das importações brasileiras provenientes dos cinco principais países de origem. É notável a queda de participação dos Estados Unidos e da Argentina, além do crescimento da participação da China e Nigéria que, por outro lado, reflete o aumento das exportações destes dois. 
TABELA 1 - TAXA DE CRESCIMENTO DOS ESTADOS BRASILEIROS E DO BRASIL: 1994-2006

\begin{tabular}{|c|c|c|c|c|c|c|c|c|c|c|c|c|c|c|}
\hline ESTADOS & 1994 & 1995 & 1996 & 1997 & $\begin{array}{c}199 \\
8\end{array}$ & $\begin{array}{c}199 \\
9\end{array}$ & $\begin{array}{c}200 \\
0\end{array}$ & $\begin{array}{c}200 \\
1\end{array}$ & $\begin{array}{c}200 \\
2\end{array}$ & 2003 & 2004 & $\begin{array}{c}200 \\
5\end{array}$ & $\begin{array}{c}200 \\
6\end{array}$ & $\begin{array}{l}\text { Média } \\
\text { Simples }\end{array}$ \\
\hline Acre & 1,57 & $\underline{4,13}$ & 3,81 & 1,46 & 4,06 & 3,60 & $\underline{4,25}$ & 4,53 & 4,78 & 4,13 & 7,33 & 6,70 & 5,40 & 4,29 \\
\hline Alagoas & 7,17 & $-1,74$ & 1,29 & 5,19 & 2,66 & $-\overline{0}$ & $\underline{1,93}$ & 1,72 & $\underline{0,49}$ & $-0,72$ & $\underline{4,40}$ & 4,27 & 4,40 & $\underline{2,38}$ \\
\hline Amazonas & $\begin{array}{c}23,6 \\
9\end{array}$ & $\begin{array}{c}22,5 \\
8\end{array}$ & $\begin{array}{c}14,8 \\
0\end{array}$ & $\begin{array}{c}11,0 \\
4\end{array}$ & 1,15 & 2,92 & 9,30 & 4,17 & 7,90 & 4,41 & $\begin{array}{c}10,2 \\
7\end{array}$ & 9,67 & $\underline{2,60}$ & 9,58 \\
\hline Amapá & $\underline{5,08}$ & 4,52 & 5,02 & 5,41 & $\underline{0,54}$ & 2,39 & 5,66 & 5,72 & 5,95 & 7,83 & 7,88 & 5,80 & 5,80 & 5,12 \\
\hline Bahia & $\underline{3,59}$ & $\underline{1,02}$ & 2,68 & 6,56 & 1,69 & 2,18 & $\underline{3,89}$ & $\underline{0,99}$ & $\underline{0,99}$ & 2,14 & 9,35 & 4,61 & $\underline{2,70}$ & 3,26 \\
\hline Ceará & 6,26 & 1,52 & 3,93 & 3,62 & 2,14 & 1,69 & $\underline{4,01}$ & $=$ & 2,74 & 1,60 & $\underline{4,75}$ & $\underline{2,70}$ & 8,00 & 3,22 \\
\hline Distrito Federal & $\underline{2,88}$ & $\underline{3,52}$ & 2,62 & 4,46 & 2,84 & 3,16 & $\underline{3,23}$ & 2,50 & 2,68 & 1,80 & $\underline{4,94}$ & 4,71 & 5,40 & 3,44 \\
\hline Espírito Santo & 6,70 & $\underline{0,43}$ & 6,03 & $\underline{1,74}$ & 2,85 & 2,68 & 7,00 & $\underline{0,44}$ & 5,79 & 1,48 & 5,72 & 4,01 & 7,70 & 4,04 \\
\hline Goiás & $\underline{2,71}$ & $\underline{1,84}$ & 3,67 & 5,27 & 2,19 & 3,17 & 5,11 & 4,32 & 4,67 & 4,24 & $\underline{4,91}$ & 3,96 & $\underline{3,10}$ & 3,78 \\
\hline Maranhão & $\underline{2,27}$ & $\underline{1,55}$ & 4,60 & $\underline{2,05}$ & $\bar{z}, 51$ & 4,37 & 5,05 & 1,91 & $\underline{2,42}$ & 4,29 & 8,97 & 7,09 & 5,00 & 3,39 \\
\hline Minas Gerais & 5,55 & $\underline{3,16}$ & 5,42 & 3,66 & $\overline{0,59}$ & 1,62 & 5,09 & $\underline{0,09}$ & $\underline{2,61}$ & 1,31 & 5,85 & 3,82 & 3,90 & 3,28 \\
\hline Mato Grosso do Sul & $\underline{3,39}$ & 4,96 & 3,36 & 4,33 & 3,17 & 2,43 & $\underline{2,06}$ & 8,10 & $\underline{2,00}$ & 7,97 & $\underline{-2,21}$ & $\underline{3,06}$ & 5,20 & 3,68 \\
\hline Mato Grosso & $\begin{array}{c}11,8 \\
9\end{array}$ & $\underline{1,95}$ & 3,97 & 5,58 & 7,21 & 8,67 & 7,84 & 6,69 & 9,11 & 3,72 & $\begin{array}{c}16,0 \\
2\end{array}$ & 5,12 & $\underline{\overline{4,60}}$ & 6,40 \\
\hline Pará & $\underline{-1,60}$ & $\underline{0,03}$ & 1,10 & $\underline{2,90}$ & 2,21 & 4,71 & 5,14 & 4,66 & 3,60 & 6,15 & 7,11 & 4,05 & $\overline{7,10}$ & 3,63 \\
\hline Paraíba & $\begin{array}{c}12,9 \\
6\end{array}$ & $\underline{4,01}$ & 3,08 & $\underline{3,24}$ & $\begin{array}{c}= \\
\underline{3,42}\end{array}$ & 5,37 & 6,70 & $\underline{0,60}$ & 4,52 & 5,26 & $\underline{2,64}$ & 3,41 & 6,70 & 4,24 \\
\hline Pernambuco & $\underline{3,60}$ & $\underline{4,38}$ & $\underline{0,33}$ & 4,06 & $\overline{\bar{z}} \overline{50}$ & 1,55 & 5,04 & 1,81 & 3,73 & $\underline{-0,64}$ & $\underline{4,08}$ & 3,82 & 5,10 & $\underline{2,80}$ \\
\hline Piauí & 7,64 & $\underline{1,98}$ & 4,68 & $\underline{0,89}$ & $\overline{1,75}$ & 3,25 & $\underline{3,27}$ & $\underline{1,25}$ & $\underline{0,72}$ & 5,69 & 5,81 & 4,55 & 6,10 & 3,66 \\
\hline Paraná & 5,51 & $\underline{1,78}$ & 7,24 & $\underline{0,89}$ & 5,30 & 2,32 & 5,06 & 4,59 & $\underline{1,46}$ & 4,62 & $\underline{4,64}$ & $\overline{0,26}$ & $\underline{2,00}$ & 3,47 \\
\hline Rio de Janeiro & $\underline{2,38}$ & $\underline{2,54}$ & $\underline{2,03}$ & $\underline{1,08}$ & 2,52 & 1,76 & $\underline{2,89}$ & $\underline{1,23}$ & 4,67 & $\underline{-0,98}$ & $\underline{3,26}$ & $\underline{2,73}$ & 4,00 & $\underline{2,31}$ \\
\hline $\begin{array}{l}\text { Rio Grande do } \\
\text { Norte }\end{array}$ & $\begin{array}{c}10,0 \\
6\end{array}$ & 4,63 & 3,06 & 6,27 & 2,29 & 3,20 & $\underline{4,10}$ & 1,36 & $\underline{2,30}$ & 1,49 & $\underline{3,59}$ & 3,59 & 4,80 & 3,90 \\
\hline Rondônia & 8,78 & $\underline{3,34}$ & 4,00 & $\underline{1,15}$ & $\underline{\overline{0}, \overline{75}}$ & 5,46 & $\underline{4,30}$ & 6,54 & 9,13 & 5,46 & 9,01 & 4,38 & $\underline{3,60}$ & 4,95 \\
\hline Roraima & $\underline{3,54}$ & 4,74 & 4,28 & 3,46 & $\overline{\overline{0}} \overline{43}$ & 4,80 & 4,49 & 5,87 & 5,95 & 3,48 & 5,75 & 4,23 & 6,30 & 4,34 \\
\hline Rio Grande do Sul & $\underline{5,19}$ & $\underline{-5,01}$ & $\underline{0,47}$ & 6,06 & $\underline{\overline{0.53}}$ & 3,00 & 4,44 & 3,03 & $\underline{1,22}$ & 1,80 & $\underline{3,05}$ & $\underline{\overline{3,00}}$ & 4,70 & $\underline{1,88}$ \\
\hline Santa Catarina & $\underline{3,34}$ & 5,77 & 4,11 & 5,25 & $\overline{0,58}$ & 3,83 & 4,62 & 3,88 & $\underline{1,39}$ & 1,18 & 7,32 & 1,41 & $\underline{2,60}$ & 3,48 \\
\hline Sergipe & $\underline{2,40}$ & $\underline{1,03}$ & 4,31 & 7,18 & 0,88 & 1,28 & $\underline{3,28}$ & $\underline{0,30}$ & 3,22 & 2,77 & 6,46 & 5,20 & 4,10 & 3,26 \\
\hline São Paulo & 5,94 & 4,68 & 2,65 & 3,84 & 0,31 & 0,36 & $\underline{3,70}$ & $\underline{1,18}$ & $\underline{0,82}$ & $\underline{-0,30}$ & $\underline{5,87}$ & $\underline{3,44}$ & $\underline{4,00}$ & $\underline{2,81}$ \\
\hline Tocantins & $\underline{2,68}$ & $\underline{4,34}$ & 3,60 & 3,92 & 3,94 & 3,28 & $\underline{3,65}$ & $\underline{0,42}$ & $\underline{2,54}$ & $\begin{array}{c}10,2 \\
9\end{array}$ & 7,41 & 6,93 & $\underline{3,10}$ & 4,31 \\
\hline Brasil & 5,33 & 4,42 & 2,15 & 3,38 & 0,04 & 0,25 & 4,31 & 1,31 & 2,66 & 1,15 & 5,71 & 3,16 & 3,97 & 2,91 \\
\hline
\end{tabular}

FONTES: Para 2002-2005: Sistema de Contas Regionais Referência 2002. Para 1985-2001: Antigo Sistema de Contas Regionais, sendo ambas do IBGE. As estimativas do PIB a custo de fatores e a preços básicos são iguais à soma dos setores: agropecuária, industria e serviços. PIB Brasil - IBGE/SCN 2000 Anual - SCN_PIBG

NOTA: Números sublinhados representam uma taxa de crescimento abaixo da média nacional, enquanto que os demais são aqueles com uma taxa de crescimento acima da média nacional 
GRÁFICO 3 - EVOLUÇÃO DAS IMPORTAÇÕES BRASILEIRAS

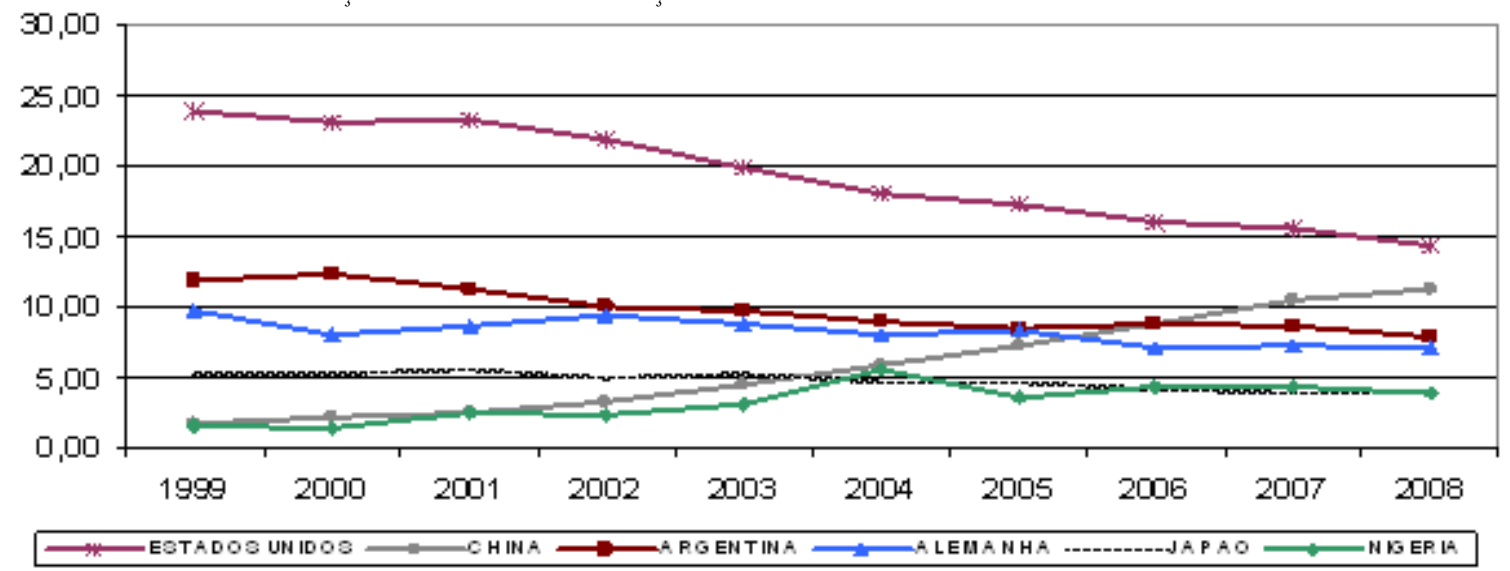

FONTE: Elaboração própria a partir dos dados do SECEX/MDIC

Pelo Gráfico 4 a elevação das importações provenientes da China também fica evidente para o caso paranaense. Outro país com destaque é a Nigéria, assim como para o Brasil. Podemos notar ainda uma queda relativa considerável das importações paranaenses com origem na Alemanha, Estados Unidos e Argentina.

GRÁFICO 4 - EVOLUÇÃO DAS IMPORTAÇÕES PARANAENSES

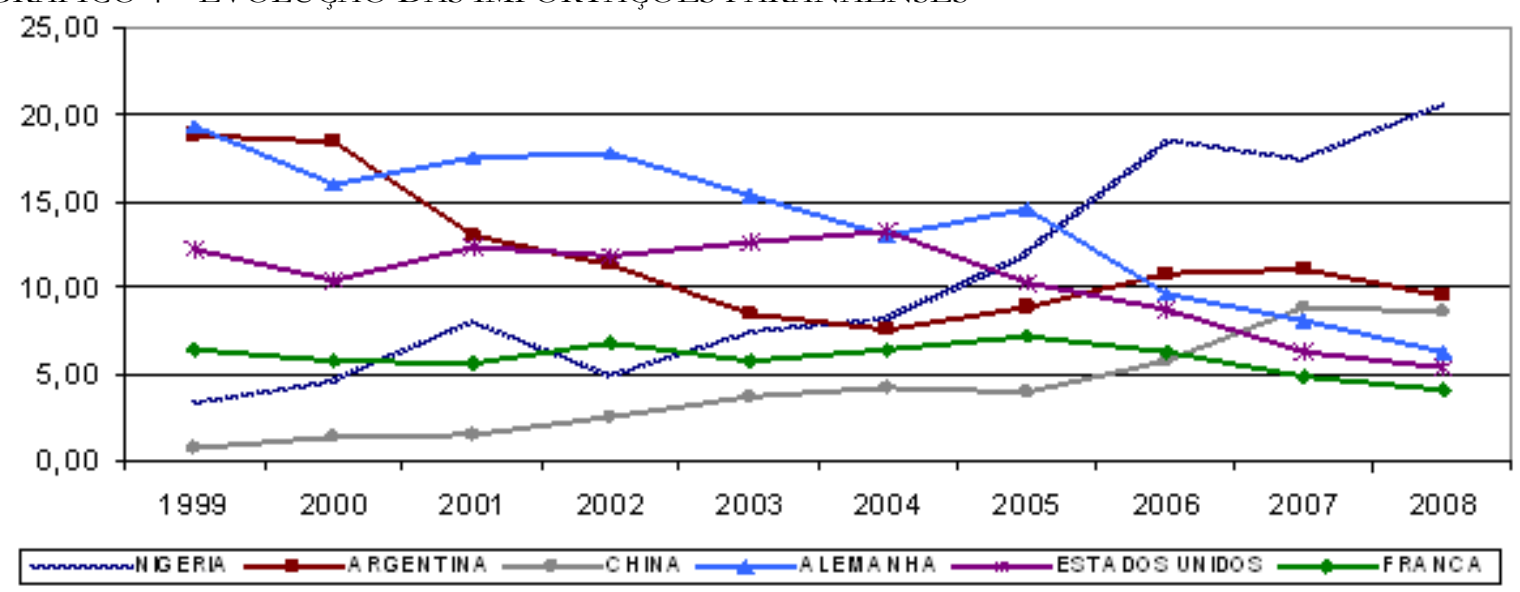

FONTE: Elaboração própria a partir dos dados do SECEX/MDIC

Os dados para os 30 principais países que exportam para o Paraná estão na Tabela 2.

De uma forma geral, percebe-se uma queda das importações provenientes da América do Norte e Europa e uma elevação das importações com origem nos países asiáticos e africanos.

TABELA 2 - EVOLUÇÃO DAS IMPORTAÇÕES PARANAENSES: 30 PRINCIPAIS PAÍSES

\begin{tabular}{|c|c|c|c|c|c|c|c|c|c|c|}
\hline PAÍSES & 1999 & 2000 & 2001 & 2002 & 2003 & 2004 & 2005 & 2006 & 2007 & 2008 \\
\hline NIGERIA & 3,39 & 4,58 & 8,17 & 4,95 & 7,47 & 8,20 & 12,01 & 18,56 & 17,34 & 20,64 \\
\hline ARGENTINA & 18,82 & 18,44 & 13,06 & 11,33 & 8,56 & 7,64 & 8,87 & 10,78 & 11,06 & 9,55 \\
\hline CHINA & 0,71 & 1,46 & 1,49 & 2,53 & 3,68 & 4,24 & 3,98 & 5,77 & 8,94 & 8,67 \\
\hline ALEMANHA & 19,34 & 15,93 & 17,58 & 17,79 & 15,31 & 12,98 & 14,53 & 9,62 & 8,09 & 6,26 \\
\hline ESTADOS UNIDOS & 12,19 & 10,40 & 12,35 & 11,90 & 12,58 & 13,32 & 10,36 & 8,75 & 6,28 & 5,43 \\
\hline FRANÇA & 6,49 & 5,86 & 5,73 & 6,78 & 5,78 & 6,48 & 7,23 & 6,27 & 4,86 & 4,15 \\
\hline RUSSIA, FEDERAÇÃO DA & 1,55 & 2,26 & 1,89 & 2,46 & 3,62 & 4,59 & 1,97 & 1,95 & 2,82 & 4,08 \\
\hline MÉXICO & 0,81 & 2,39 & 3,05 & 2,40 & 1,73 & 1,27 & 1,15 & 1,91 & 3,83 & 3,08 \\
\hline CANADÁ & 2,23 & 2,78 & 1,44 & 1,74 & 1,22 & 1,51 & 1,09 & 1,23 & 1,48 & 2,10 \\
\hline SUÉCIA & 2,23 & 2,89 & 2,50 & 2,13 & 2,43 & 2,61 & 2,77 & 1,99 & 2,40 & 1,98 \\
\hline ITÁLIA & 4,40 & 4,14 & 5,63 & 3,45 & 3,87 & 3,74 & 3,24 & 2,55 & 2,18 & 1,95 \\
\hline
\end{tabular}


MARROCOS

CHILE

PARAGUAI

ESPANHA

JAPÃO

ISRAEL

ANGOLA

TAIWAN (FORMOSA)

MALÁSIA

TAILÂNDIA

REINO UNIDO

BELARUS

INDIA

PAISES BAIXOS (HOLANDA)

CORÉIA, REPÚBLICA DA (SUL)

BÉLGICA

TCHECA, REPÚBLICA

POLONIA

SUÍCA

\begin{tabular}{llllllllll}
0,04 & 0,06 & 0,27 & 0,43 & 0,48 & 0,70 & 0,42 & 0,34 & 1,00 & 1,87 \\
1,29 & 1,79 & 1,34 & 1,64 & 2,02 & 2,43 & 2,76 & 2,77 & 2,17 & 1,87 \\
3,14 & 3,36 & 2,93 & 5,59 & 7,15 & 2,93 & 3,21 & 1,57 & 1,81 & 1,76 \\
2,72 & 1,83 & 2,60 & 1,72 & 1,79 & 2,20 & 2,55 & 1,91 & 2,45 & 1,67 \\
1,61 & 1,99 & 2,92 & 1,99 & 2,33 & 2,43 & 2,56 & 1,90 & 1,74 & 1,64 \\
2,07 & 1,94 & 2,29 & 2,93 & 2,18 & 2,87 & 1,95 & 1,96 & 1,49 & 1,55 \\
0,18 & 0,19 & 0,30 & 0,00 & 0,01 & 0,00 & 0,00 & 1,06 & 0,00 & 1,52 \\
1,14 & 0,72 & 0,75 & 1,13 & 0,67 & 0,96 & 1,24 & 1,63 & 2,06 & 1,38 \\
0,09 & 0,44 & 0,23 & 0,31 & 0,46 & 0,85 & 1,42 & 1,41 & 1,45 & 1,30 \\
0,08 & 0,07 & 0,19 & 0,25 & 0,29 & 0,40 & 0,32 & 0,40 & 0,78 & 1,14 \\
1,13 & 1,36 & 1,39 & 3,12 & 1,82 & 2,46 & 1,97 & 1,74 & 1,47 & 1,09 \\
0,07 & 0,29 & 0,33 & 0,87 & 0,74 & 0,88 & 0,68 & 0,63 & 0,63 & 1,08 \\
0,11 & 0,08 & 0,12 & 0,42 & 0,25 & 1,00 & 0,57 & 0,98 & 1,02 & 0,99 \\
0,53 & 0,55 & 0,50 & 0,80 & 0,70 & 0,72 & 0,77 & 0,71 & 0,67 & 0,72 \\
1,86 & 1,78 & 1,92 & 1,54 & 0,39 & 0,47 & 0,42 & 0,45 & 0,56 & 0,72 \\
0,96 & 0,79 & 0,69 & 1,09 & 1,17 & 1,22 & 1,03 & 0,93 & 0,75 & 0,72 \\
0,08 & 0,08 & 0,05 & 0,12 & 0,31 & 0,45 & 1,63 & 0,84 & 0,65 & 0,65 \\
0,09 & 0,20 & 0,20 & 0,17 & 0,78 & 0,94 & 0,49 & 0,82 & 0,51 & 0,61 \\
1,08 & 0,83 & 0,75 & 1,03 & 1,93 & 1,62 & 0,98 & 0,65 & 0,62 & 0,58 \\
\hline
\end{tabular}

\section{COMPOSIÇÃO DA PAUTA DE IMPORTAÇÕES PARANAENSE}

O produto que teve maior elevação na pauta de importações do estado foi óleos brutos de petróleo, com uma elevação da participação na pauta de importações paranaenses de 12,69\%, em 2005, para 24,59\%, em 2008, considerando que os dados de 2008 vão apenas até junho (Tabela 3). Adicionalmente, elevação da participação dos seguintes produtos: outros cloretos de potássio (3,19\% para 4,39\%); superfosfato, teor de pentóxido de fósforo $\left(\mathrm{P}_{2} \mathrm{O}_{5}\right)>45 \%(0,22 \%$ para 2,57\%); diidrogeno-ortofosfato de amônio, incl. mist. hidrogen. etc (1,65\% para 1,94\%); hidrogeno-ortofosfato de diamônio, teor arsênio > $=6 \mathrm{mg} / \mathrm{kg}(0,09 \%$ para 1,68\%); outs. adubos/fertiliz. miner. quim. c/ nitrogênio e fósforo ( $0 \%$ para $1 \%)$; sulfato de amônio $(0,47 \%$ para $0,68 \%)$; e adubos ou fertilizantes c/nitrogênio, fósforo e potássio $(0 \%$ para 0,52\%); entre 1999 e 2008, refletem o crescimento e a importância do setor agrícola no estado.

Dando suporte aos dados apresentados na Tabela 3, pelo Gráfico 5 fica clara a elevação das importações da Petrobrás S.A., aumento este que está relacionado aos óleos brutos de petróleo. Pelo mesmo gráfico, observa-se que a participação das importações das montadoras não se elevou, enquanto que a participação de fertilizantes foi perceptível.

Esse ponto das empresas que importam fertilizantes fica ainda mais evidente quanto analisamos os dados das 20 principais importadoras do Paraná em 2008, de acordo com os dados apresentados na Tabela 4.

\section{CONSIDERAÇÕES FINAIS}

O desempenho das importações na economia brasileira e, especificamente, no Estado do Paraná, apresentam um comportamento convergente, caracterizado por uma 
expansão significativa a partir de 2004. Essa trajetória era esperada por conta do elevado crescimento do PIB a partir de 2004, acompanhado de valorização da taxa de câmbio, entre o início de 2003 até setembro de 2008.

Junto à expansão das importações em termos agregados, o artigo chamou a atenção para alguns aspectos relevantes, relativos à origem e composição dos produtos importados. Em primeiro lugar, tanto no Brasil, como no caso específico do Paraná destacaram-se a queda das importações advindas dos Estados Unidos e Argentina, concomitantemente ao aumento de participação das importações de produtos chineses. No caso paranaense, destaca-se também o aumento de participação da Nigéria, para o qual são apresentados indícios de que isso teria sido resultado do aumento da importação de óleos brutos de petróleo pela refinaria da Petrobrás, instalada em Araucária.

TABELA 3 - EVOLUÇÃO DOS 30 PRINCIPAIS PRODUTOS IMPORTADOS PELO PARANÁ: 19992008

\begin{tabular}{|c|c|c|c|c|c|c|c|c|c|c|}
\hline PARANÁ & 1999 & 2000 & 2001 & 2002 & 2003 & 2004 & 2005 & 2006 & 2007 & 2008 \\
\hline Oleos brutos de petróleo & 8,42 & 13,0 & 11,5 & 8,87 & 9,88 & 8,20 & 12,6 & 21,9 & 18,6 & 24,5 \\
\hline $\begin{array}{l}\text { Autom c/motor expl, } 1500<\mathrm{cm} 3<=3000 \text {, até } 6 \\
\text { passag }\end{array}$ & 9,39 & 4,51 & 4,07 & 1,50 & 0,81 & 0,87 & 0,70 & 5,40 & 7,48 & 5,73 \\
\hline Outros cloretos de potássio & 3,19 & 3,22 & 2,38 & 3,76 & 3,96 & 5,36 & 3,27 & 3,08 & 2,93 & 4,39 \\
\hline Superfosf, teor de pentóxido de fósforo $\left(\mathrm{P}_{2} \mathrm{O}_{5}\right)>45 \%$ & 0,22 & 0,64 & 0,58 & 0,74 & 1,30 & 1,73 & 0,86 & 0,63 & 1,26 & 2,57 \\
\hline Diidrog-ortofos de amônio, incl. mist. hidrogen. etc & 1,65 & 1,48 & 1,68 & 1,82 & 2,64 & 3,40 & 1,17 & 0,89 & 1,61 & 1,94 \\
\hline Hidrog-ortofosf de diam, teor arsênio $>=6 \mathrm{mg} / \mathrm{kg}$ & 0,09 & 0,24 & 0,17 & 0,30 & 0,40 & 0,68 & 0,48 & 0,30 & 1,09 & 1,68 \\
\hline Outras partes e acess. de carroçarias p/veíc. autom & 2,06 & 2,57 & 2,84 & 3,61 & 3,46 & 3,02 & 2,53 & 1,90 & 1,88 & 1,54 \\
\hline Uréia com teor de nitrogênio $>45 \%$ em peso & 0,27 & 0,76 & 0,34 & 0,46 & 1,03 & 1,22 & 0,85 & 0,71 & 0,95 & 1,47 \\
\hline Outros circuitos integrados & 0,00 & 0,00 & 0,00 & 0,00 & 0,00 & 0,00 & 0,00 & 0,00 & 1,77 & 1,35 \\
\hline Trigo e trigo c/centeio & 1,69 & 1,40 & 1,05 & 1,48 & 1,97 & 0,56 & 0,56 & 0,88 & 0,79 & 1,19 \\
\hline Caixas de marchas $\mathrm{p} /$ veículos automóveis & 0,91 & 1,51 & 1,76 & 2,18 & 1,90 & 1,89 & 2,29 & 1,69 & 1,49 & 1,17 \\
\hline Outras partes e acess. p/tratores e veículos autom & 2,72 & 3,50 & 2,60 & 2,86 & 2,90 & 2,51 & 2,74 & 1,72 & 1,55 & 1,17 \\
\hline $\begin{array}{l}\text { Outs. adubos/fertiliz. miner. quím. c/nitrogênio e } \\
\text { fósforo }\end{array}$ & 0,00 & 0,02 & 0,07 & 0,43 & 0,86 & 1,11 & 0,57 & 0,55 & 0,81 & 1,00 \\
\hline Automóveis c/motor explosão, cil $<=1000 \mathrm{~cm}^{3}$ & 1,22 & 0,55 & 0,65 & 0,23 & 0,08 & 0,01 & 0,00 & 0,00 & 0,68 & 0,86 \\
\hline Metanol (álcool metílico) & 0,16 & 0,29 & 0,31 & 0,61 & 0,83 & 0,78 & 0,67 & 0,67 & 0,65 & 0,75 \\
\hline Sulfato de amônio & 0,47 & 0,61 & 0,72 & 0,60 & 0,79 & 1,11 & 0,49 & 0,57 & 0,66 & 0,68 \\
\hline Malte não torrado, inteiro ou partido & 0,44 & 0,43 & 0,50 & 0,72 & 0,19 & 0,31 & 0,16 & 0,27 & 0,62 & 0,60 \\
\hline Tela p/microcomputadores portáteis, policromática & 0,00 & 0,00 & 0,00 & 0,00 & 0,00 & 0,00 & 0,00 & 0,14 & 0,49 & 0,59 \\
\hline Unidades de discos magnéticos, $\mathrm{p} /$ discos rígidos & 0,09 & 0,04 & 0,05 & 0,06 & 0,02 & 0,11 & 0,39 & 0,60 & 0,75 & 0,56 \\
\hline Outros veíc autom $\mathrm{c} /$ motor diesel, $\mathrm{p} /$ carga $<=5 \mathrm{t}$ & 0,00 & 0,00 & 0,41 & 0,07 & 0,00 & 0,00 & 0,00 & 0,04 & 0,56 & 0,56 \\
\hline Adubos ou fert. c/nitrogênio, fósforo e potássio & 0,00 & 0,00 & 0,00 & 0,00 & 0,03 & 0,02 & 0,00 & 0,00 & 0,10 & 0,52 \\
\hline Outros mot. de expl., p/veíc. cap. 87 , sup. $1000 \mathrm{~cm}^{3}$ & 0,99 & 1,69 & 1,48 & 2,12 & 1,44 & 0,61 & 1,81 & 0,75 & 0,68 & 0,45 \\
\hline Farinha de trigo & 0,13 & 0,10 & 0,13 & 0,15 & 0,01 & 0,03 & 0,03 & 0,15 & 0,42 & 0,43 \\
\hline Milho em grão,exceto para semeadura & 0,31 & 0,23 & 0,41 & 0,32 & 0,56 & 0,33 & 0,49 & 0,59 & 0,82 & 0,42 \\
\hline Acido fosfonom. e ac. trimetilfosfonico & 0,00 & 0,00 & 0,00 & 0,00 & 0,12 & 0,33 & 0,12 & 0,06 & 0,24 & 0,41 \\
\hline Injetores para motores diesel ou semidiesel & 0,05 & 0,09 & 0,12 & 0,24 & 0,32 & 0,44 & 0,48 & 0,61 & 0,63 & 0,40 \\
\hline Outs. Comp. heter.. c/1 ciclo pirazol, $\mathrm{n} /$ condens. & 0,00 & 0,00 & 0,00 & 0,61 & 0,00 & 0,00 & 0,00 & 0,00 & 0,47 & 0,40 \\
\hline Outs. partes de bombas p/líquidos & 0,00 & 0,00 & 0,00 & 0,00 & 0,00 & 0,00 & 0,00 & 0,66 & 0,50 & 0,38 \\
\hline Outras unidades de discos ópticos & 0,01 & 0,02 & 0,01 & 0,01 & 0,01 & 0,07 & 0,16 & 0,33 & 0,44 & 0,36 \\
\hline "Gasóleo" (oleo diesel) & 0,00 & 0,00 & 0,00 & 0,62 & 0,38 & 1,61 & 0,00 & 0,57 & 0,53 & 0,33 \\
\hline Óleos brutos de petróleo & 8,42 & 13,0 & 11,5 & 8,87 & 9,88 & 8,20 & 12,6 & 21,9 & 18,6 & 24,5 \\
\hline
\end{tabular}

FONTE: Elaboração própria a partir de dados do SECEX/MDIC.

NOTAS: os dados 2008 são até junho. Os 30 principais produtos são classificados de acordo com sua ordem de importância em 2008 
GRÁFICO 5 - EVOLUÇÃO DAS IMPORTAÇÕES DAS 6 (SEIS) PRINCIPAIS EMPRESAS IMPORTADORAS DO PARANÁ - 2008

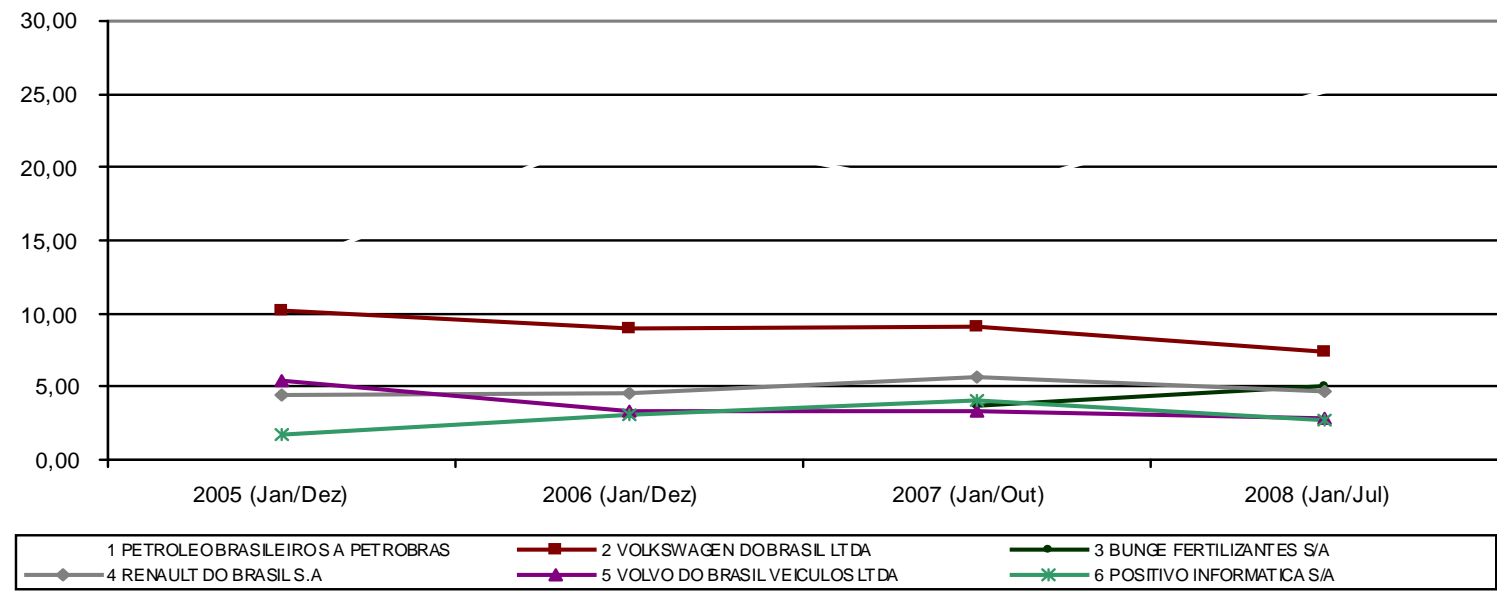

FONTE: SECEX/MDIC

TABELA 4 - EVOLUÇÃO DAS IMPORTAÇÕES DAS 20 (VINTE) PRINCIPAIS EMPRESAS IMPORTADORAS DO PARANÁ, 2008

\begin{tabular}{|c|c|c|c|c|c|}
\hline & EMPRESAS & $\begin{array}{c}2005 \\
\text { (Jan/Dez) }\end{array}$ & $\begin{array}{c}2006 \\
\text { (Jan/Dez) }\end{array}$ & $\begin{array}{c}2007 \\
\text { (Jan/Out) }\end{array}$ & $\begin{array}{c}2008 \\
\text { (Jan/Jul) }\end{array}$ \\
\hline 1 & PETRÓLEO BRASILEIRO S A PETROBRÁS & 12,87 & 22,88 & 18 & 25,21 \\
\hline 2 & VOLKSWAGEN DO BRASIL LTDA & 10,24 & 8,98 & 9,12 & 7,32 \\
\hline 3 & BUNGE FERTILIZANTES S/A & --- & --- & 3,73 & 5,10 \\
\hline 4 & RENAULT DO BRASIL S.A & 4,45 & 4,59 & 5,71 & 4,64 \\
\hline 5 & VOLVO DO BRASIL VEÍCULOS LTDA & 5,37 & 3,34 & 3,28 & 2,81 \\
\hline 6 & POSITIVO INFORMÁTICA S/A & 1,71 & 3,10 & 4,05 & 2,75 \\
\hline 7 & MOSAIC FERTILIZANTES DO BRASIL S/A & --- & --- & 1,53 & 2,42 \\
\hline 8 & NISSAN DO BRASIL AUTOMÓVEIS LTDA & 1,81 & 1,18 & 3,01 & 2,20 \\
\hline 9 & CNH LATIN AMERICA LTDA & 1,39 & 1,45 & 2,11 & 1,87 \\
\hline 10 & FERTIPAR FERTILIZANTES DO PARANÁ LIMITADA & --- & --- & 1,03 & 1,35 \\
\hline 11 & ROBERT BOSCH LIMITADA & 4,18 & 2,54 & 1,81 & 1,29 \\
\hline 12 & SADIA S.A. & 1,34 & 1,57 & 1,23 & 1,20 \\
\hline 13 & $\begin{array}{l}\text { MACROFERTIL - INDÚSTRIA E COMERCIO DE } \\
\text { FERTILIZANTES }\end{array}$ & 0,61 & 0,83 & 0,68 & 1,02 \\
\hline 14 & FERTILIZANTES HERINGER S.A. & 0,40 & 0,49 & 0,7 & 0,95 \\
\hline 15 & YARA BRASIL FERTILIZANTES S/A & & & 0,54 & 0,94 \\
\hline 16 & COOPERATIVA AGRÍCOLA CENTRO-OESTE LTDA & 0,67 & 0,68 & 0,67 & 0,85 \\
\hline 17 & MILENIA AGROCIÊNCIAS S.A. & --- & 0,93 & 1,19 & 0,78 \\
\hline 18 & DFV - TELECOMUNICAÇÕES E INFORMÁTICA S.A. & & & & 0,74 \\
\hline 19 & ADM DO BRASIL LTDA & --- & --- & 0,41 & 0,70 \\
\hline 20 & NORTOX SA & 0,45 & 0,39 & 0,67 & 0,69 \\
\hline
\end{tabular}

FONTE: SECEX/MDIC

Por fim, com relação à pauta de importações paranaense, observou-se que nos últimos anos a mudança mais significativa ocorreu por conta do aumento de participação da importação de óleo brutos de petróleo, o qual teve um aumento de preço expressivo no mercado internacional a partir de 2003, sendo ainda mais intensivo entre 2006 e o primeiro semestre de 2008.

Portanto, apesar de algumas mudanças significativas na composição da pauta de importações do Paraná após 2004, observou-se que parte expressiva deste comportamento 
pode ser explicada pelo aumento dos preços internacionais de petróleo. Entretanto, desde o início do segundo semestre de 2008, tem-se observado uma queda significativa nos preços internacionais desta commodity ${ }^{42}$, além da queda da expectativa de crescimento da economia brasileira e paranaense em 2009. Esses dois efeitos tendem a reduzir a pressão sobre as pautas de importações brasileira e paranaense. Por outro lado, no curto prazo, a tendência é que a pressão se mantenha por conta da desvalorização abrupta da taxa de câmbio. No médio prazo, tanto por conta da desvalorização da taxa de câmbio, como da provável desaceleração da economia brasileira e da queda dos preços das commodities internacionais, haverá uma tendência a uma queda das importações brasileiras e paranaenses.

\section{REFERÊNCIAS}

BANCO CENTRAL DO BRASIL. Relatório Focus. Brasília, 12/12/2008. Disponível em: $<$ http://www4.bcb.gov.br/pec/GCI/PORT/readout/R20081212.pdf $>$. Acesso em $15 / 12 / 2008$.

IPEADATA. Brasília: IPEA. Disponível em: <http://www.ipeadata.gov.br>. Acesso em $15 / 12 / 2008$.

\section{ANEXO}

GRÁFICO 6 - PIB - VARIAÇÃO REAL ANUAL (1999-2008)

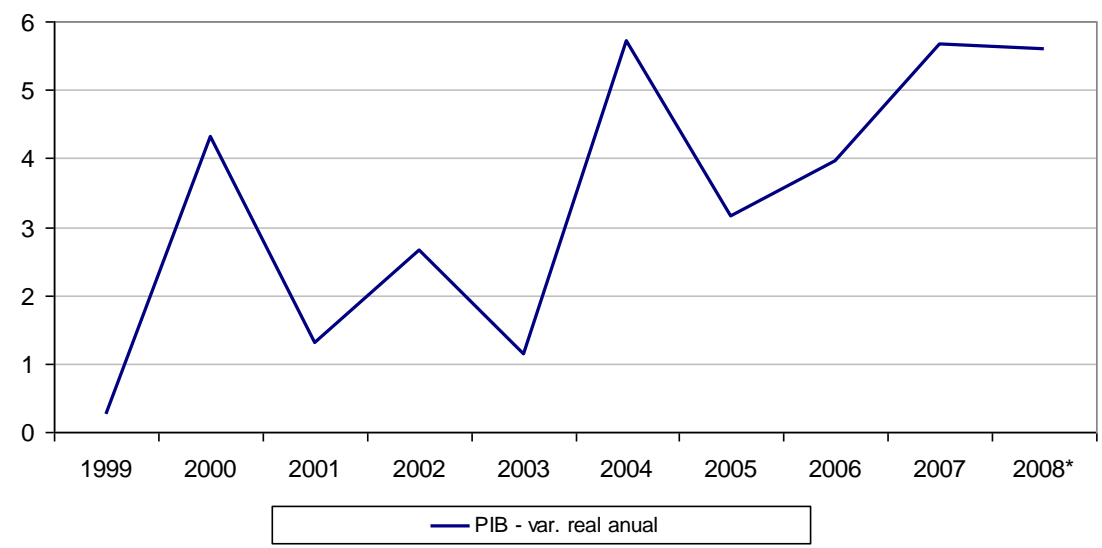

Fonte: SCN/IBGE apud IPEADATA (2008a)

NOTA: para o ano de 2008 está sendo apresentada uma projeção com base nas expectativas de mercado, disponíveis no relatório Focus do Banco Central do Brasil, publicado em 12 de dezembro de 2008.

GRÁFICO 7 - TAXA DE CÂMBIO NOMINAL - (1998-2008)

42 ver anexo: Gráfico 9. 


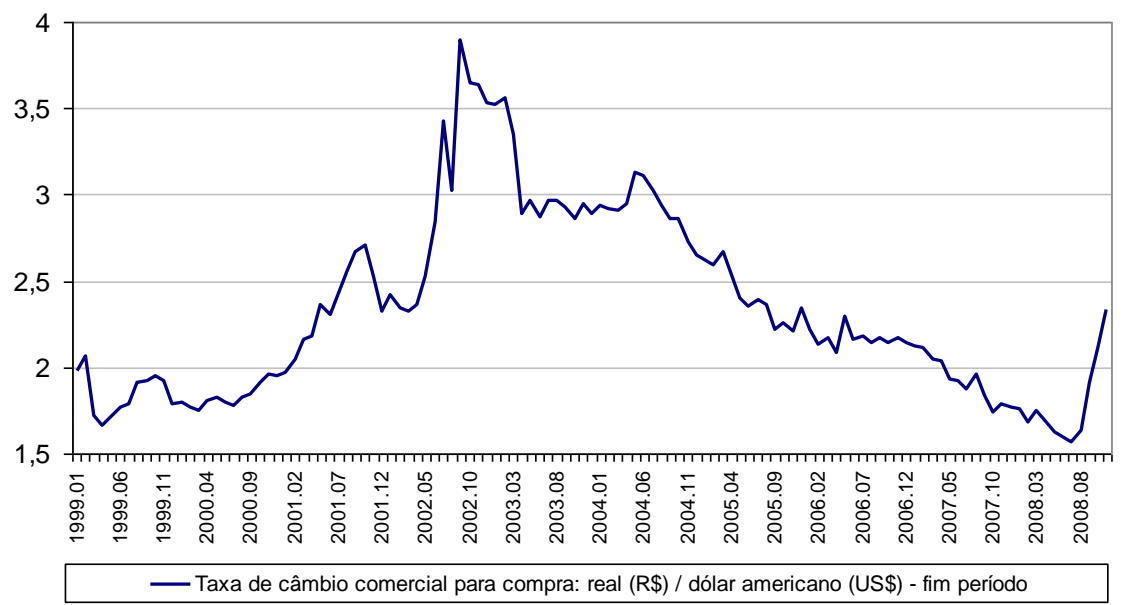

FONTE: Banco Central do Brasil apud IPEADATA (2008b).

GRÁFICO 8 - TAXA DE CÂMBIO EFETIVA REAL - (1998-2008)

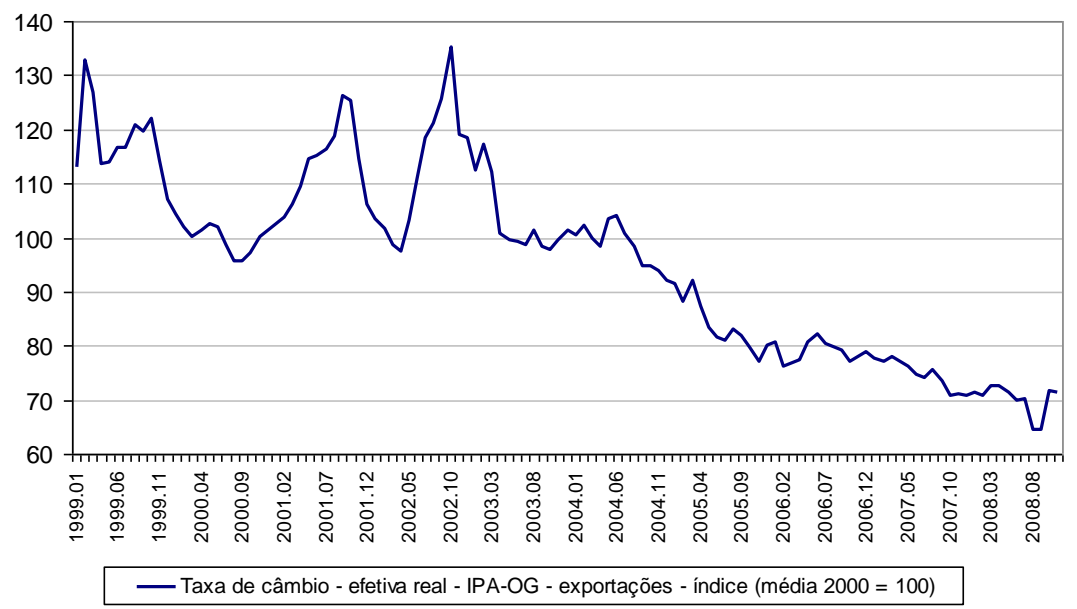

FONTE: Banco Central do Brasil apud IPEADATA (2008).

GRÁFICO 9 - ÍNDICE DE PREÇOS INTERNACIONAIS DE PETRÓLEO E DERIVADOS - (1998-2008)

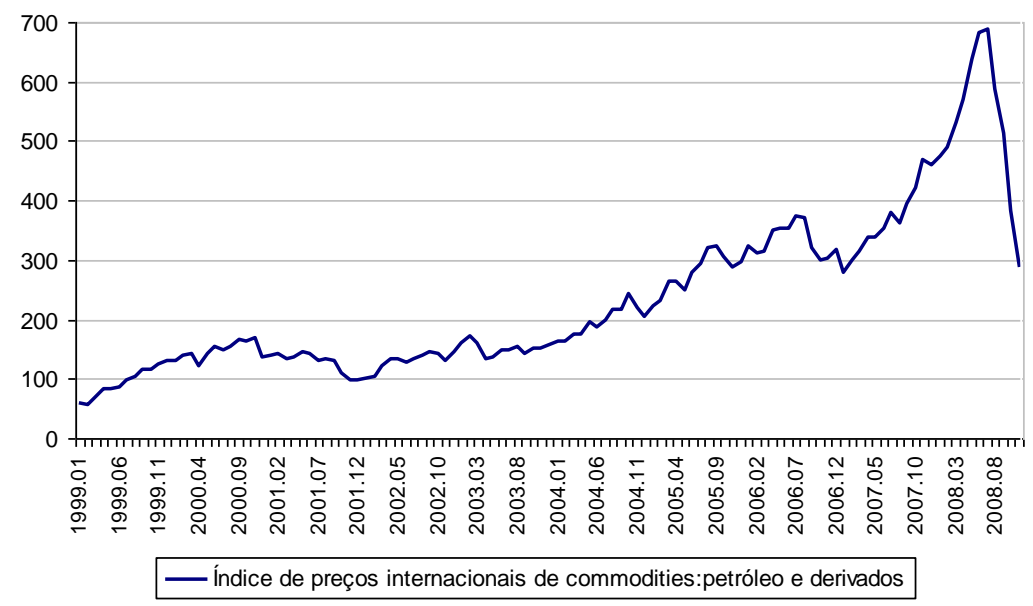

FONTE: IPEA apud IPEADATA (2008). 